SLAC-PUB-9731

May 2003

\title{
Kaluza-Klein/ $Z^{\prime}$ Differentiation at the LHC and Linear Collider *
}

\author{
Thomas.G. Rizzo ${ }^{\dagger}$ \\ Stanford Linear Accelerator Center, Stanford, CA, 94309
}

\begin{abstract}
We explore the capabilities of the LHC and the Linear Collider(LC) to distinguish the production of Kaluza-Klein(KK) excitations from an ordinary $Z^{\prime}$ within the context of theories with $\mathrm{TeV}$ scale extra dimensions. At the LHC, these states are directly produced in the Drell-Yan channel while at the LC the effects of their exchanges are indirectly felt as new contact interactions in processes such as $e^{+} e^{-} \rightarrow f \bar{f}$. While we demonstrate that the $\mathrm{LC}$ is somewhat more capable at $\mathrm{KK} / Z^{\prime}$ differentiation than is the LHC, the simplest LC analysis relies upon the LHC data for the resonance mass as an important necessary input.
\end{abstract}

*Work supported by the Department of Energy, Contract DE-AC03-76SF00515

${ }^{\dagger}$ e-mail: rizzo@slac.stanford.edu 


\section{Introduction}

The possibility of KK excitations of the Standard Model(SM) gauge bosons within the framework of theories with TeV-scale extra dimensions has been popular for some time[1]. The proven variety of such models is very large and continues to grow. For example, given the possibility of warped or flat extra dimensions one can construct a large number of interesting yet distinct models whose detailed structure depends upon a number of choices, e.g., whether all the gauge fields experience the same number of dimensions, whether the fermions and/or Higgs bosons are also in the bulk, whether brane kinetic terms are important[2] in the determination of the KK spectrum and couplings and whether there exists a conservation law of KK number or KK parity, as in the case if of the Universal Extra Dimensions(UED)[3] scenario. If such particles do exist how will they be observed at colliders and how will we know that we have observed signals for extra dimensions and not some other new physics signature? For example, it is well known that UED can mimic supersymmetry at the LHC[3] unless the spins of the new KK states can be measured.

In the analysis below we will be interested in the question of distinguishing the lightest KK excitations of the SM electroweak gauge bosons from a more conventional $Z^{\prime}$ at both the LHC and LC. At the LHC single $\mathrm{KK} / Z^{\prime}$ production is most easily observed via the DrellYan mechanism whereas, at the LC, the exchange of either set of states leads to contact interaction-like modifications to processes such as $e^{+} e^{-} \rightarrow f \bar{f}$. This question already limits our focus to a rather specific class of theories and excludes many others. For example, at the tree level in UED, a conserved KK-parity exists which forbids the single production or exchange of KK states by zero modes and thus this class of theories is clearly excluded from our considerations. In addition we can exclude models whose couplings and spectra are such that multiple KK resonances will be directly observable at the LHC. In this case there can be no issue of confusion as to whether or not extradimensional signatures are being produced. We also can exclude from consideration the set of models wherein the KK excitations of only the $S U(2)_{L}$ or $U(1)_{Y}$ gauge bosons can be produced. If either of these possibilities were realized and the spectrum of the KK fields was such that second and higher resonances were beyond the reach of the LHC, one can easily convince oneself that the $\mathrm{KK}$ and $Z^{\prime}$ interpretations cannot be distinguished. A similar situation holds for $W^{ \pm} \mathrm{KK}$ excitations alone even when the entire $S U(2)_{L} \times U(1)_{Y}$ gauge structure is in the bulk. Of course after making these few cuts in model space many theories remain. 
The simplest model of the class we will consider is the case of only one flat extra dimension where all the fermions are constrained to lie at one of the two orbifold fixed points, $y=0, \pi R$, associated with the compactification on $S^{1} / Z_{2}$ [4], where $R$ is the radius of the compactified extra dimension. Under usual circumstances a 3-brane is located at each of the fixed points upon which ordinary 4-d fields will reside. In principle, a SM fermion can be localized on the brane at either fixed point consistent with the constraints of gauge invariance. In our discussions below we will consider two specific cases: either all of the fermions are placed at $y=0(D=0)$ or the quarks and leptons are localized at opposite fixed points $(D=\pi R)$. Here $D$ is the distance between the quarks and leptons in the single extra dimension. (The later model may be of interest in the suppression of proton decay in certain schemes. Certainly more complicated scenarios are possible even if we assume generation independence.) In such schemes the fermionic couplings of the KK excitations of a given gauge field are identical to those of the SM apart from a possible sign if the fermions live at the $y=\pi R$ fixed point and an overall factor of $\sqrt{2}$. The gauge boson KK excitation masses are given, to lowest order in $\left(M_{0} / M_{c}\right)^{2}$, by the relationship $M_{n}^{2}=\left(n M_{c}\right)^{2}+M_{0}^{2}$, where $n$ labels the KK level, $M_{c}=1 / R \sim 1 \mathrm{TeV}$ is the compactification scale and $M_{0}$ is the zero-mode mass obtained via spontaneous symmetry breaking for the cases of the $W$ and $Z$. Here we have assumed that any brane localized kinetic terms which may be present[2] do not significantly alter these naive results. Note that the first KK excitations of the photon and $Z$ will be highly degenerate in mass, becoming more so as $M_{c}$ increases. For example, if $M_{c}=4 \mathrm{TeV}$ the splitting between the first $Z$ and $\gamma \mathrm{KK}$ states is less than $\sim 1 \mathrm{GeV}$, too small to be observed at the LHC. An updated analysis[4] of precision electroweak data implies that $M_{c} \geq 4-5 \mathrm{TeV}$, independently of whether the Higgs field is in the bulk or on the brane and upon which of the fixed points the various SM fermions are confined. This is a range directly accessible to the LHC for resonance production. Interestingly, at a LC with a center of mass energy of $\sqrt{s}=500-1000 \mathrm{GeV}$ the effects of $\mathrm{KK}$ exchanges with masses well in excess of the 4-5 TeV range are also easily observable as is shown in Fig. 1.

Of course this large value of $M_{c}$ implies that the LHC experiments will at best observe only a single bump in the $\ell^{+} \ell^{-}$channel as the next set of KK states, which are essentially twice as heavy, 8-10 TeV, are too massive to be seen even with an integrated luminosity of order $1-3 a b^{-1}[5]$. (Such high luminosities may be approachable at an upgrade of the LHC[11].) This can be seen from Fig. 2 which assumes for simplicity that all fermions lie at either the $y=0$ or $y=\pi R$ fixed points, i.e., $D=0$ or $\pi R$. These apparently isolated 
single resonance structures are actually a superposition of the individual excitations of both the SM $\gamma$ and $Z$ which are highly degenerate as we noted above. It is this dual excitation plus the existence of additional tower states that lead to the very unique resonance shape in either case. Note that above the first KK resonance the excitation curves for the $D=0$ and $\pi R$ cases are essentially identical. This figure shows that KK states up to masses somewhat in excess of $\simeq 7 \mathrm{TeV}$ or so should be directly observable at the LHC or the LHC with a luminosity upgrade in a single lepton pair channel.

It is important to note that if brane terms are important then the bounds on $M_{c}$ from precision measurements can be significantly weaker due to reduced fermion-KK gauge couplings thus allowing for a much lighter first KK state. However it may then be possible to directly observe the higher excitations so that no confusion with $Z^{\prime}$ production would occur. However, parameter space regions may exist where such a possibility can be avoided; such scenarios are beyond the scope of the present analysis.

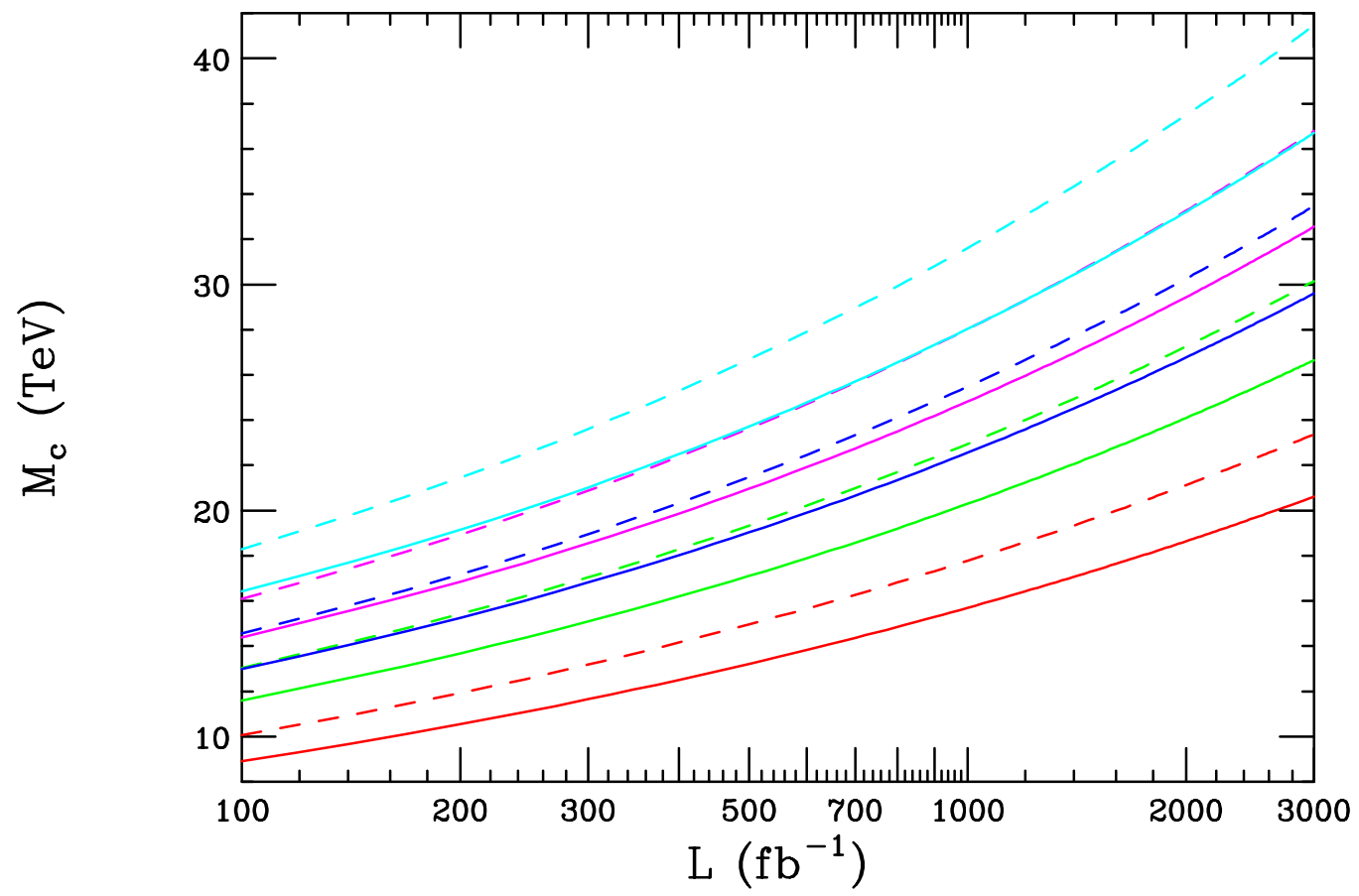

Figure 1: 95\% CL bound on the scale $M_{c}$ as a function of the LC integrated luminosity from the reaction $e^{+} e^{-} \rightarrow f \bar{f}$, where $f=\mu, \tau, c, b, t$ have been summed over. The solid(dashed) curves assume a positron polarization $P_{+}=0(0.6)$; an electron polarization of $80 \%$ has been assumed in all cases. From bottom to top the center of mass energy of the LC is taken to be $0.5,0.8,1,1,2$ and $1.5 \mathrm{TeV}$, respectively.

If a gauge KK resonance structure is observed in Drell-Yan, how will this observation 

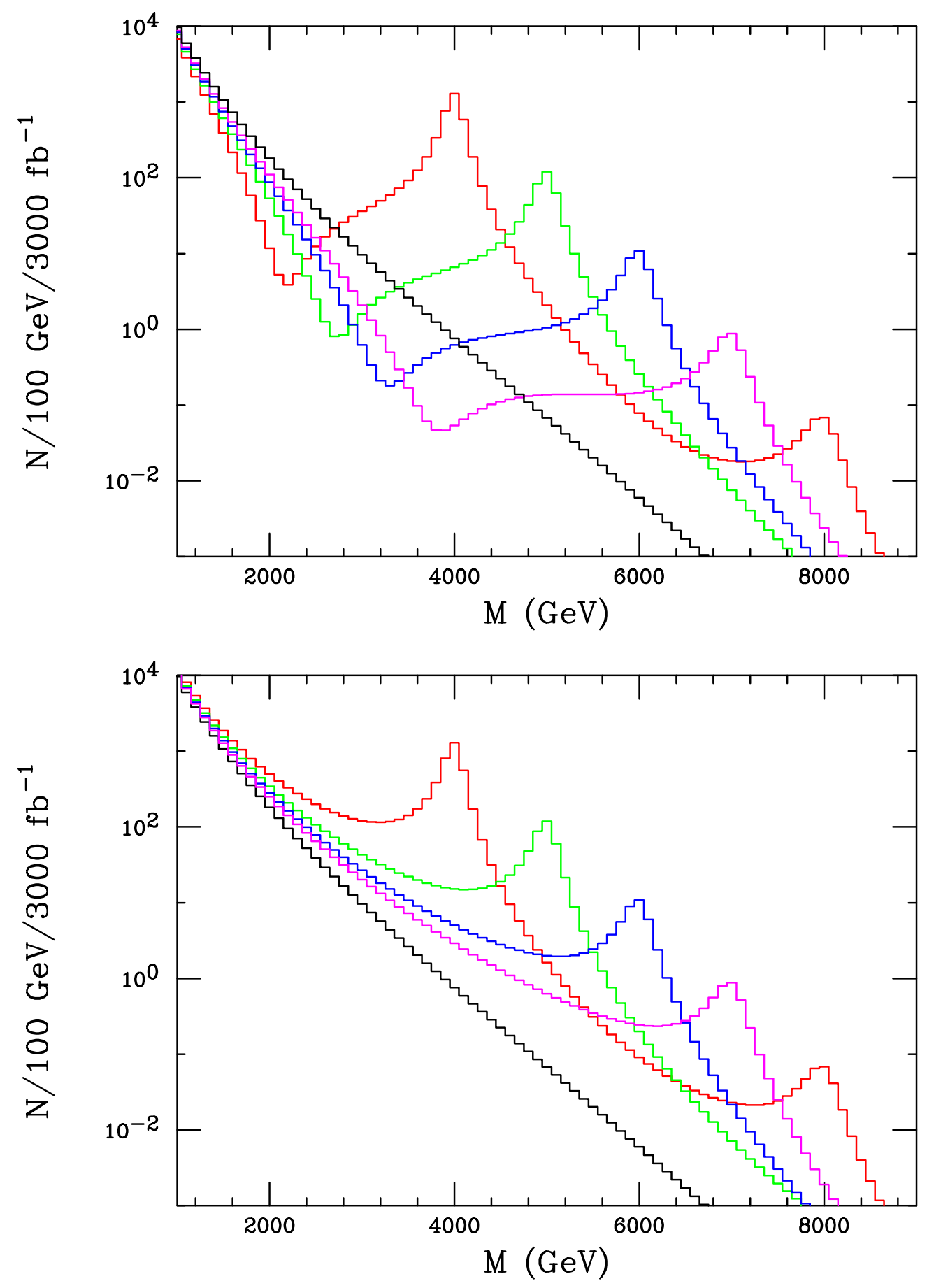

Figure 2: Production rate, in the Drell-Yan channel $p p \rightarrow e^{+} e^{-} X$, for $\gamma / Z \mathrm{KK}$ resonances as a function of dilepton invariant mass assuming a very high luminosity LHC. A rapidity cut $\left|\eta_{l}\right| \leq 2.5$ has been applied to the final state leptons. The red(green, blue, magenta) histogram corresponds $M_{c}=4(5,6,7) \mathrm{TeV}$, respectively. The black histogram is the SM background. In the top panel all fermions are assumed to lie at the $y=0$ fixed point, $D=0$, while the quarks and leptons are split, $D=\pi R$, in the lower panel. 
be interpreted? (Here we imagine a time line where the LC turns on after several years of data taking by the LHC at roughly the time of an LHC luminosity upgrade.) Through straightforward measurement of the lepton pair angular distribution it will be known immediately that the resonance is spin-1 and not, e.g., a spin-2 graviton resonance as in the Randall-Sundrum[6] model[7], provided sufficient luminosity is available. In addition, the existence of an essentially degenerate pair of resonances in both the charged and neutral Drell-Yan channels will forbid a possible graviton interpretation. Perhaps the most straightforward possibility for interpretation would be that of an extended gauge model[8] which predicts the existence of a degenerate $W^{\prime}$ and $Z^{\prime}$; many such models already exist in the literature[9]. Is it possible to distinguish this degenerate $Z^{\prime} / W^{\prime}$ model from KK excitations without seeing the rest of the tower? Clearly, based on the discussion above, we must focus on differentiating the $Z^{\prime}$ from the first (and only observable) KK excitation spectra below and around the peak. At least temporarily, only LHC data will be available for this discrimination until the LC subsequently turns on.

\section{What Can the LHC Tell Us?}

The issue of $\mathrm{KK} / Z^{\prime}$ differentiation at the LHC has been previously discussed to a limited extent by several authors in Ref.[10]. The purpose of this section is to generalize those analyses as well as to extend them to the case of higher integrated luminosities and eventually make comparisons with the LC. Though more than this earlier work, the present analysis much will still leave much that remains to be studied. Pictorially we will consider the case $M_{c}=4 \mathrm{TeV}$ but our analysis will be extended to significantly larger mass values.

Fig. 3 shows a closeup of the excitation spectra and forward-backward asymmetries, $A_{F B}$, for KK production near the first resonance region assuming $M_{c}=4 \mathrm{TeV}$ and with $D=0, \pi R$. There are several comments to be made at this point. First, for $p p$ colliders, the forward-backward asymmetry is defined via the angle made by the direction of the negatively charged lepton and the direction of motion of the center of mass in the laboratory frame. This direction is assumed to be the same as that of the initial state quark, which is reasonable given the harder valence parton distribution. Second, we observe the by now familiar strong destructive interference minimum[1] in the cross section for the $D=0$ case near $M \simeq 0.55 M_{c}$ which is also reflected in the narrow dip in the asymmetry. This dip structure is a common feature that will persist even in higher dimensional models or in models with warped extra 
dimensions. The precise location of the dip is sensitive to model details, however. Third, we notice that the overall behaviour of the $D=0$ and $D=\pi R$ cases is completely different. In fact, if anything, the $D=\pi R$ case displays a strong constructive interference in the region below the KK peak. This difference in the two excitation curves is due solely to the additional factor of $(-1)^{n}$ appearing in the KK sum arising from the placement of the quarks and leptons at opposite fixed points. (Here, $n$ labels the KK number of the state.) Lastly, we note that the peak cross section and peak $A_{F B}$ values, respectively, are nearly identical in the two cases. In the narrow width approximation the two sets of values are identical since the additional sign factors are found to cancel.

For either $D$ choice the excitation curve and $A_{F B}$ appear to be qualitatively different than that which one obtains for typical $Z^{\prime}$ models[8] as is shown in Figs. 4 and 5 . The resonance structure for the KK case is significantly wider and has a larger peak cross section than does the typical $Z^{\prime}$ model where the strong destructive interference below the resonance is absent. (We remember however that the height and width of the $Z^{\prime}$ or KK resonance also depends on the set of allowed decay modes.) In addition, the dip in the value of $A_{F B}$ occurs much closer to the resonance region for the typical $Z^{\prime}$ model than it does in the KK case. Clearly the KK resonance does not look like one of the usual $Z$ 's but we certainly could not claim, based on these figures, that some $Z^{\prime}$ model with which we are not familiar cannot mimic either KK case. In fact, from the figures, one can more easily imagine a $Z^{\prime}$ with stronger than typical couplings leading to an excitation structure similar to the $D=\pi R$ case, i.e., it seems more likely that the case of $D=\pi R$ can be mimicked by a (strongly coupled) $Z^{\prime}$ than does the $D=0$ case.

In order to quantify the differences between the KK and $Z^{\prime}$ scenarios we must choose observables that have reasonable statistical power associated with them and do not explicitly depend on any assumptions about how the $\mathrm{KK}$ or $Z^{\prime}$ may decay, i.e., if the resonance has non-SM decays. Consider the $D=0$ case; given the $100-3000 \mathrm{fb}^{-1}$ luminosity of the LHC and its upgrade the invariant mass distribution will only be useful as an observable for lepton pair masses above the $Z$ pole and below $\sim 2.5 \mathrm{TeV}$ and as well as near the $\mathrm{KK} / Z^{\prime}$ resonance. The later region we cannot use fairly if we assume that all of the decays of the resonance are a priori unknown. Note that significantly beyond the peak region the cross section is quite small yielding too poor a set of statistics to be valuable. Since the statistics required to determine $A_{F B}$ is significantly higher (because angular distributions now need to be measured as well), the range of usefulness as a differentiating observable is even more 

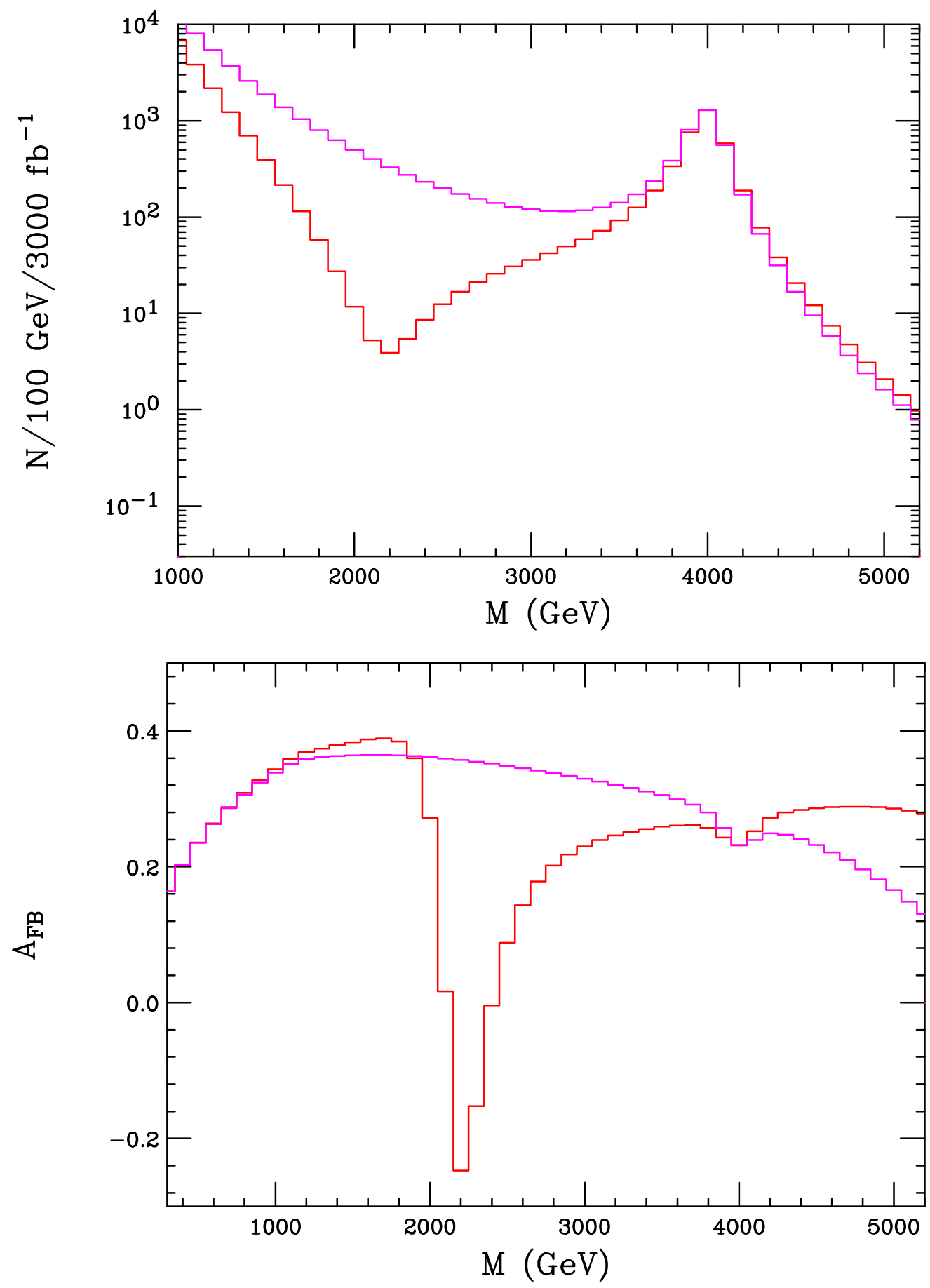

Figure 3: A comparison of the lepton pair invariant mass spectrum and forward-backward lepton asymmetry for the production of a $4 \mathrm{TeV} \mathrm{KK}$ resonance for the two choices of $D$. The red(magenta) histograms are for the case $D=0(\pi R)$. 

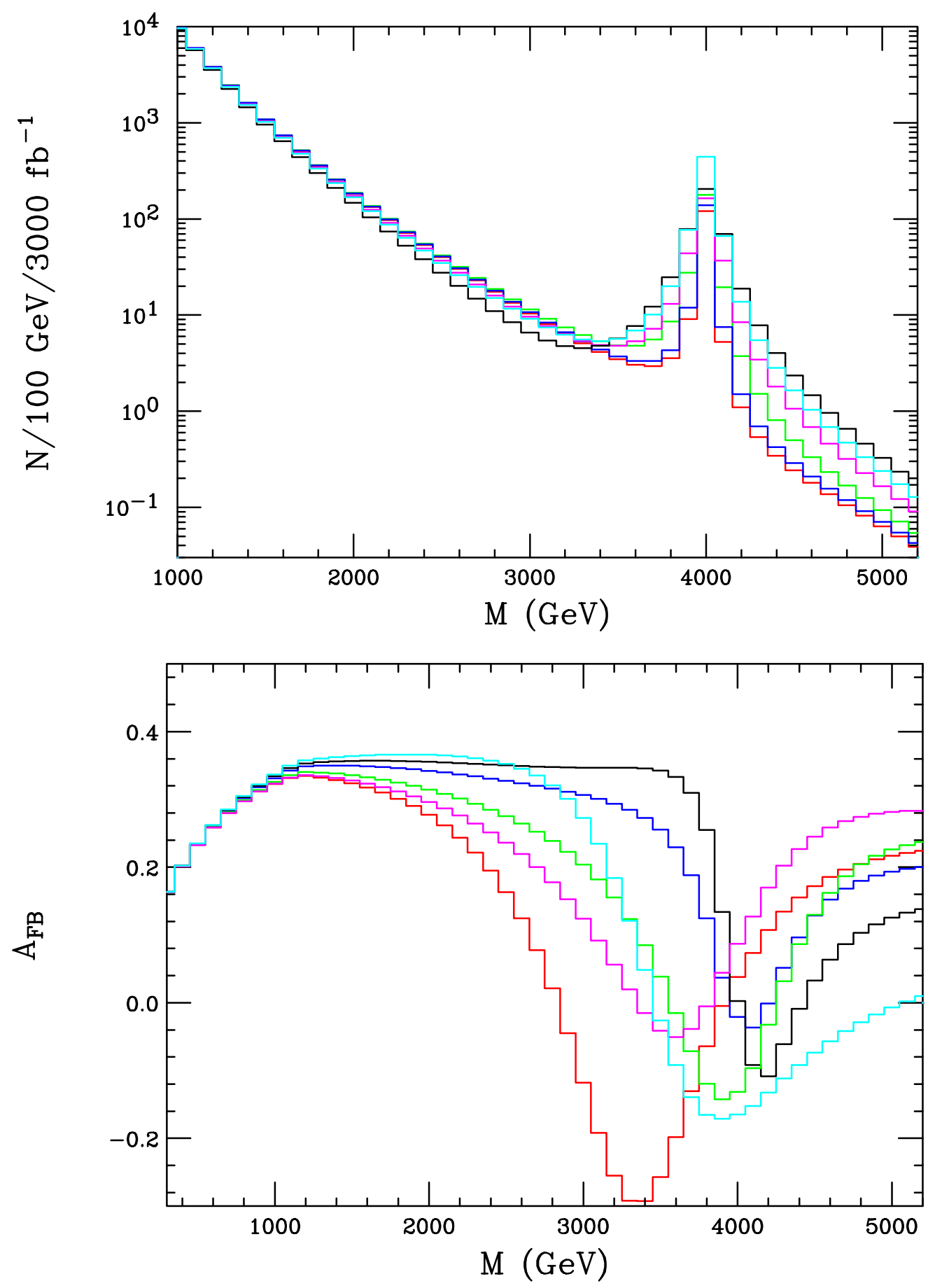

Figure 4: Same as in the previous pair of figures but now for a number of $Z^{\prime}$ models. The red(green, blue, magenta, cyan, black) histograms correspond to $E_{6}$ model $\psi(\chi, \eta)$, the Left Right Symmetric Model with $\kappa=g_{R} / g_{L}=1$, the Alternative Left Right Model and the Sequential Standard Model, respectively. For descriptions of these models and original references see Ref.[8]. 

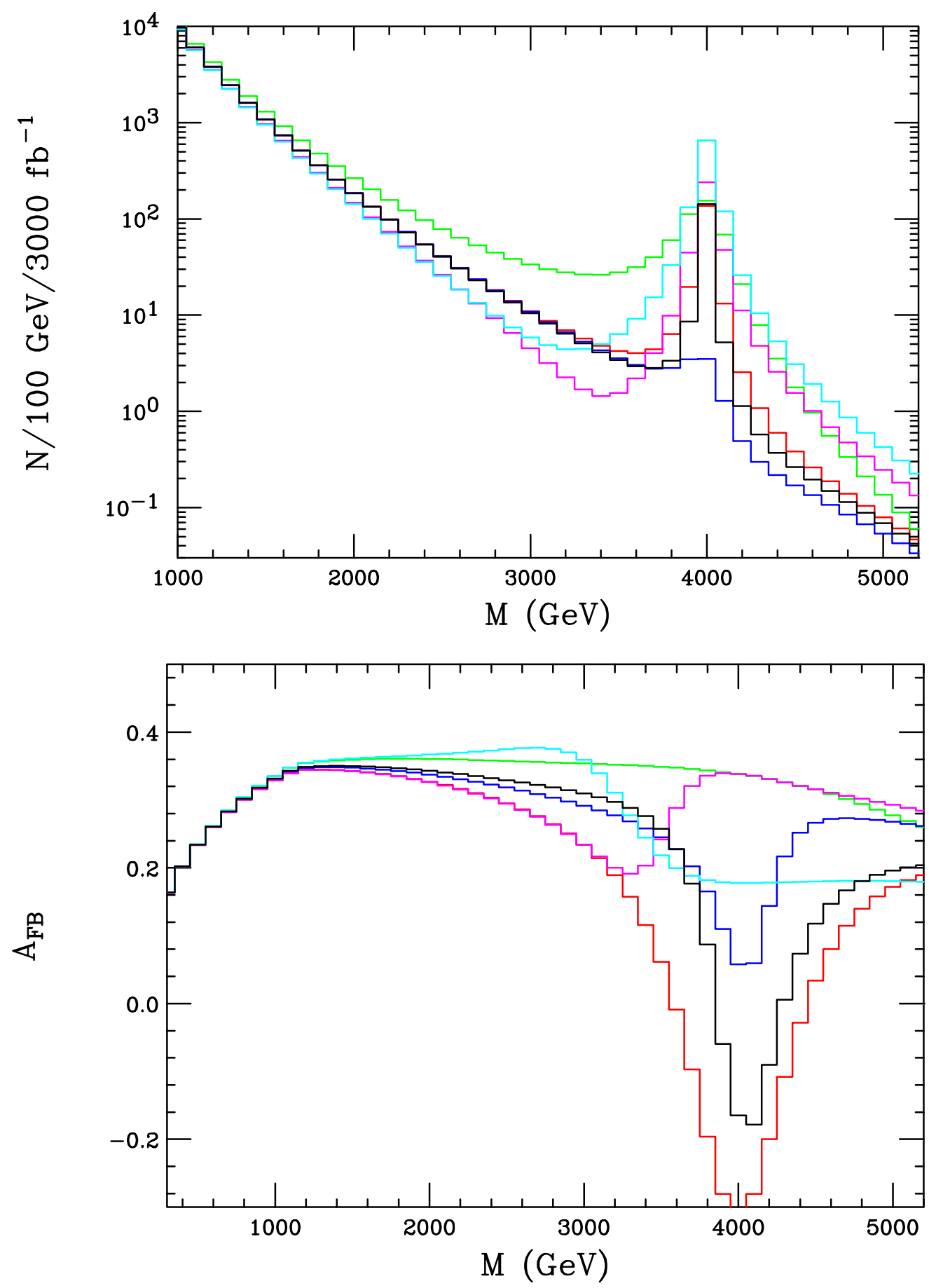

Figure 5: Same as in the previous pair of figures but now for a further set of $Z^{\prime}$ models. The red(green, blue, magenta, cyan, black) histograms correspond to $E_{6}$ model $I$, the Un-unified Model with $s_{\phi}=0.6$, the Foot-Hernandez model, the model of Kuo et al., a $Z^{\prime}$ coupling proportional to hypercharge and $E_{6}$ model $\eta$ with gauge kinetic mixing parameter $\delta=0.25$, respectively. For descriptions of these models and original references see Ref.[8]. 
restricted, perhaps below $\sim 1.5 \mathrm{TeV}$ in dilepton invariant mass. However, one might imagine that $A_{F B}$ may also be helpful very near the peak region since we know that for the case of a $Z^{\prime}$ it is approximately independent of what other modes the resonance may decay into, unlike the cross section. Naively, we would expect the inclusion of input from $A_{F B}$ data on the peak to improve the results obtained below. However, it has been shown in our earlier work[10] that even near the apparent KK pole, $A_{F B}$ depends on the relative widths of the individual $\gamma$ and $Z$ KK excitations, which is model sensitive. In the analysis presented below we will ignore any additional guidance that may arise from considering the values of $A_{F B}$ near the peak and at lepton pair masses below $\sim 1.5 \mathrm{TeV}$ and examine only the invariant mass distribution, i.e., the possible additional information obtainable from $A_{F B}$ will be ignored in the present analysis and will be left for later study.

Turning to our analysis, for $M_{c}=4(5,6) \mathrm{TeV}$ we begin by generating cross section 'data' corresponding to dilepton masses in the range 250-1850(2150, 2450) GeV in $100 \mathrm{GeV}$ bins for both the $D=0$ and $\pi R$ cases. To go any lower in mass would not be very useful as we are then dominated by either the $Z$ peak or the photon pole. For larger masses the cross section is either too small in the $D=0$ case or is dominated by the heavy resonance. Next we try to fit these cross section distributions making the assumption that the data is generated by a single $Z^{\prime}$. For simplicity, we restrict our attention to the class of $Z^{\prime}$ models with generation-independent couplings and where the $Z^{\prime}$ is associated with a new gauge group generator that commutes with weak isospin. These conditions are satisfied, e.g., by GUTinspired $Z^{\prime}$ models as well as by many others in the literature[8]. If these constraints hold then the $Z^{\prime}$ couplings to all SM fermions can be described by only 5 independent parameters: the couplings of the left-handed quark and lepton doublets and the corresponding ones for the right-handed quarks and leptons. We then vary all of these couplings independently in order to obtain the best $\chi^{2} / d f$ fit to the dilepton mass distribution and obtain the relevant probability/confidence level(CL) using statistical errors only. In practise this is a fine-grained scan over a rather large volume of the 5 -d parameter space examining more than $10^{10}$ coupling combinations for each of the cases we consider to obtain the best fit. In performing this fit it is assumed that the apparent $Z^{\prime}$ mass is the same as that of the produced KK state which will be directly measured. In this approach, the overall normalization of the cross section is determined at the $Z$-pole which is outside of the fit region and is governed solely by SM physics.

The results of performing these fits for different values of $M_{c}$ and the two choices 

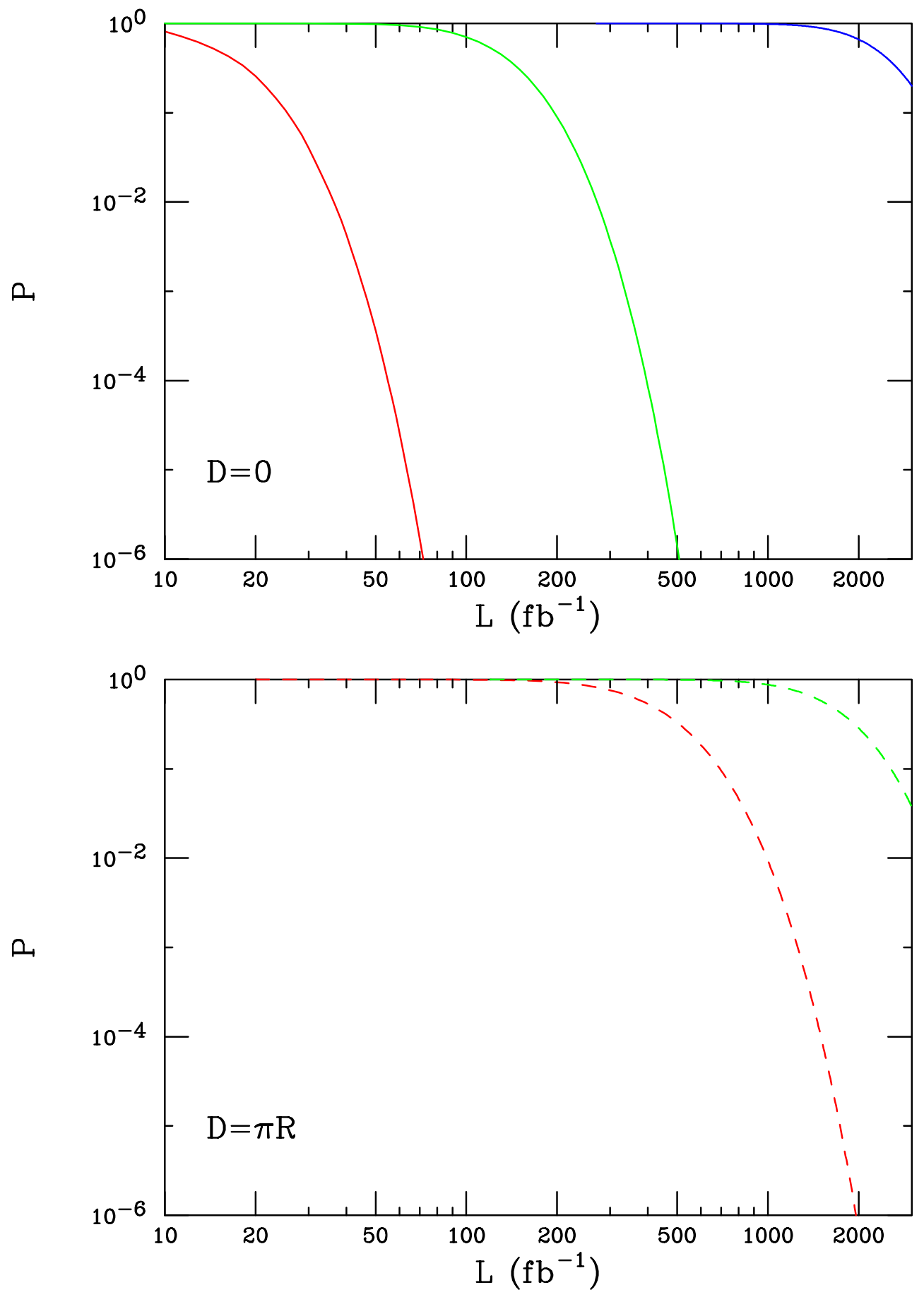

Figure 6: Probability associated with the best $Z^{\prime}$ fit hypothesis as a function of the LHC integrated luminosity for the cases $D=0$ and $D=\pi R$. From left to right the curves correspond to the choice $M_{c}=4,5$ and $6 \mathrm{TeV}$, respectively. 
$D=0, \pi R$ are shown in Fig. 6. Explicitly, these show the best fit probability for the $Z^{\prime}$ hypothesis to the KK generated data. For example, taking the case $D=0$ with $M_{c}=4$ $\mathrm{TeV}$ we see that with an integrated luminosity of order $60 \mathrm{fb}^{-1}$ the best fit probability is near a few $\times 10^{-5}$. For such low probabilities we can certainly claim that the KK generated 'data' is not well fit by the $Z^{\prime}$ hypothesis. As the mass of the KK state increases the size of the shift in the production cross section from the SM expectation is reduced and greater statistics are needed to obtain the same probability level. For $M_{c}=5 \mathrm{TeV}$ we see that an integrated luminosity of order $400 \mathrm{fb}^{-1}$ is required to get to the same level of rejection of the $Z^{\prime}$ hypothesis. Similarly, for $M_{c}=6 \mathrm{TeV}$ extremely high luminosities of order 7-8 $a b^{-1}$ would be required to get to this level of probability, which is most likely beyond even that expected for the LHC upgrades.

For the $D=\pi R$ case we see the situation is somewhat different in that the level of 'confusion' between the KK and $Z^{\prime}$ is potentially greater. This is what we might have expected based on our discussion above. Even for the case $M_{c}=4 \mathrm{TeV}$ we see that only at very high integrated luminosities of order $\sim 1.5 a b^{-1}$ can the KK and $Z^{\prime}$ scenarios be distinguished. With $M_{c}=5 \mathrm{TeV}$, approximately $6 a b^{-1}$ would be required to do the same job. For larger KK masses this separation becomes essentially impossible at the LHC.

\section{What Can the LC Tell Us?}

The analysis for the LC is somewhat different than at the LHC. No actual resonances are produced but deviations from SM cross sections and asymmetries are observed due the $s$ channel exchanges of the $Z^{\prime}$ or KK gauge boson towers. Though subtle these two sets of deviations are not identical and our hope here is to use the precision measurement capability of a LC to distinguish them. We will assumed that data is taken at a single value of $\sqrt{s}$ so that the mass of the KK or $Z^{\prime}$ resonance obtained from the LHC must be used as an input to the analysis as presented here. Without such an input the analysis below can still be performed provided data from at least two distinct values of $\sqrt{s}$ are used as input[12]. In that case $M_{c}$ becomes an additional fit parameter to be determined by the analysis from

the $\sqrt{s}$ dependence of the deviations from the SM expectations. While this more general situation is certainly very interesting it is beyond the scope of the present analysis.

Consider the general process $e^{+} e^{-} \rightarrow f \bar{f}$; assuming KK states are actually present with a fixed $M_{c}$ as above, we generate 'data' for both the differential cross section as well 

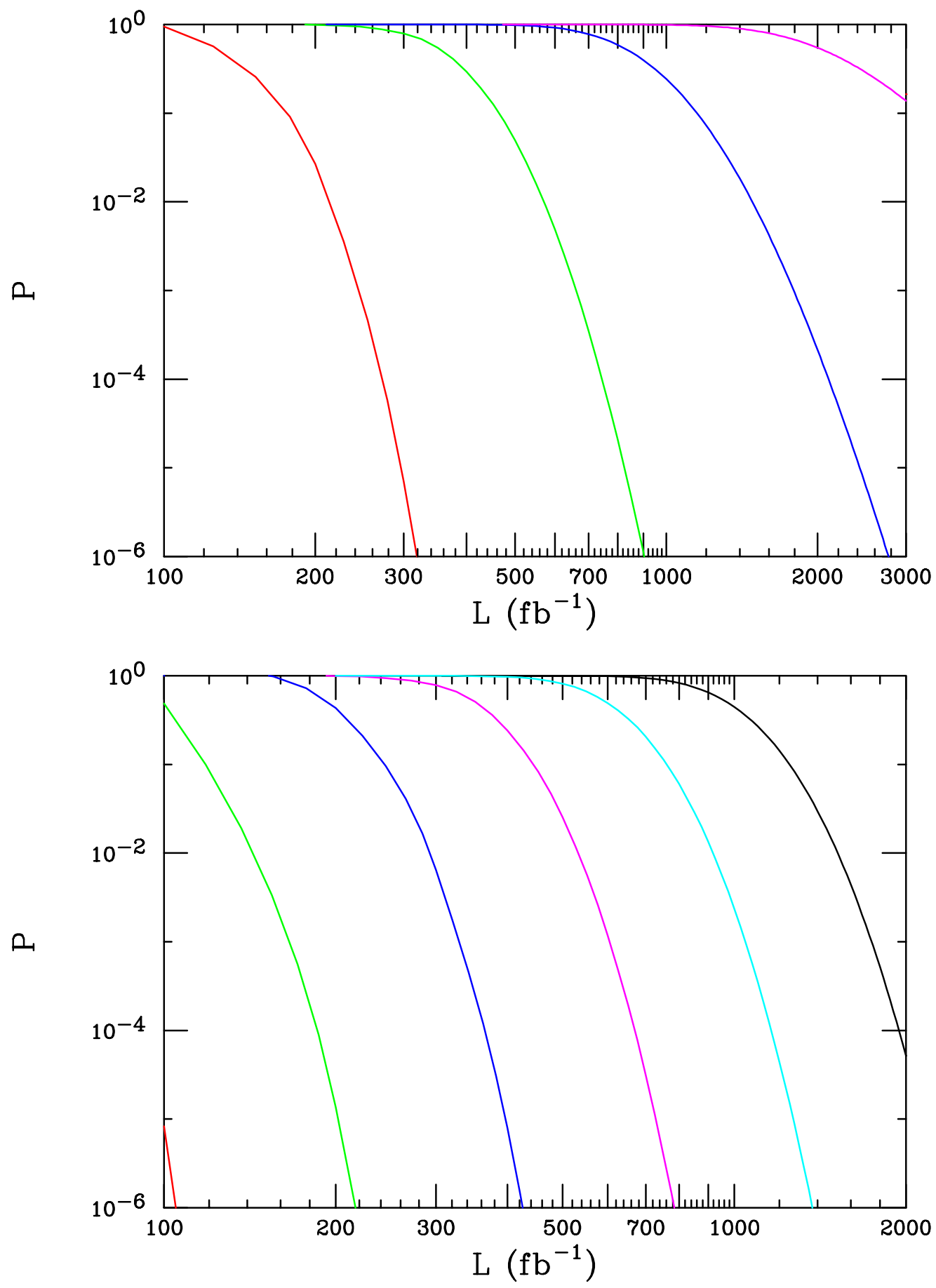

Figure 7: Same as the previous figure but now for a 500(1000) GeV LC in the top(bottom) panel. The $D=0$ and $D=\pi R$ cases are identical here. From left to right the curves are for the cases $M_{c}=4,5,6, . . \mathrm{TeV}$ etc. The value of $M_{c}$ is assumed to be determined at the LHC. 
as the Left-Right polarization asymmetry, $A_{L R}$, including the effects of ISR, as functions of the scattering angle, i.e., $\cos \theta$, in 20 (essentially) equal sized bins. The electron beam is assumed to be $80 \%$ polarized and angular acceptance cuts are applied. Our other detailed assumptions in performing this analysis are the same as those employed in earlier studies and can be found in Ref.[13]. We then try to fit this 'data' making the assumption that the deviations from the SM are due to a single $Z^{\prime}$. For simplicity, here we will concentrate on the processes $e^{+} e^{-} \rightarrow \mu^{+} \mu^{-}, \tau^{+} \tau^{-}$as only the two leptonic couplings are involved in performing any fits. In this case the $D=0$ and $D=\pi R$ scenarios lead to identical results for the shifts in all observables at the LC, an advantage over the LHC case. Adding new final states, such as $b \bar{b}$ or $c \bar{c}$, may lead to potential improvements although additional fit parameters now must be introduced and the $D=0$ and $D=\pi R$ predictions would then again be distinct as at the LHC. To be specific, we consider two cases for the LC center of mass energy: $\sqrt{s}=0.5$ and $1 \mathrm{TeV}$.

As before in the LHC case we next vary the two assumed $Z^{\prime}$ couplings to leptons to obtain the best $\chi^{2} / d f$ for the fit which then leads to the probabilities shown in Fig. 7. For the case of a $\sqrt{s}=500 \mathrm{GeV}$ LC, we see that an integrated luminosity of $300 \mathrm{fb}^{-1}$ is roughly equivalent to $60 \mathrm{fb}^{-1}$ at the LHC for the case of $M_{c}=4 \mathrm{TeV}$ assuming $D=0$. For larger values of $M_{c}$ the $500 \mathrm{GeV}$ LC does a slightly better job at $\mathrm{KK} / Z^{\prime}$ discrimination: 800(2200) $\mathrm{fb}^{-1}$ at the LC equivalent is found to be roughly equivalent to 400(7500) $\mathrm{fb}^{-1}$ at the LHC assuming $M_{c}=5(6) \mathrm{TeV}$. Since the $D=0$ and $\pi R$ cases are identical at the LC a further advantage is obtained there as noted earlier. Once the LC energy increases to $1 \mathrm{TeV}$ the LC is seen to be superior in model separation but the analysis still relies upon the LHC to input the value of $M_{c}$ in the fits. The lower panel in Fig. 7 shows results for values of $M_{c}$ beyond the range of 7-8 TeV which is directly observable at the LHC. This seems to imply that by extending the present analysis to include input from at least two values of $\sqrt{s}$ we may be able to extend the $\mathrm{KK} / Z^{\prime}$ separation out to very large masses at the LC.

\section{Summary and Conclusion}

New physics signatures arising from different sources may be confused when first observed at future colliders. Thus it is important to examine how various scenarios may be differentiated given the availability of only limited information. In this analysis we have performed a 
comparison of the capabilities of the LHC and LC to differentiate new physics associated with $\mathrm{KK}$ and $Z^{\prime}$ excitations. In the present study the LC reach was found to be somewhat superior to that of the LHC but the LC analysis depended upon the LHC determination of the resonance mass as an input. It would be useful to perform both of these studies at the level of fast $\mathrm{MC}$ to verify the results obtained here, including the input of $A_{F B}$ data at the LHC and Bhabha scattering at the LC. The analysis as presented here can also be extended to other scenarios

\section{Acknowledgements}

The author would like to thank G. Azuelos, J. Hewett, and G. Polesello for discussions related to the this analysis.

\section{References}

[1] See, for example, I. Antoniadis, Phys. Lett. B246, 377 (1990); I. Antoniadis, C. Munoz and M. Quiros, Nucl. Phys. B397, 515 (1993); I. Antoniadis and K. Benalki, Phys. Lett. B326, 69 (1994)and Int. J. Mod. Phys. A15, 4237 (2000); I. Antoniadis, K. Benalki and M. Quiros, Phys. Lett. B331, 313 (1994).

[2] M. Carena, E. Ponton, T. M. Tait and C. E. Wagner, arXiv:hep-ph/0212307; M. Carena, T. M. Tait and C. E. Wagner, Acta Phys. Polon. B 33, 2355 (2002) [arXiv:hepph/0207056]; H. Davoudiasl, J. L. Hewett and T. G. Rizzo, arXiv:hep-ph/0212279.

[3] T. Appelquist, H. C. Cheng and B. A. Dobrescu, Phys. Rev. D 64, 035002 (2001) [arXiv:hep-ph/0012100]; H. C. Cheng, K. T. Matchev and M. Schmaltz, Phys. Rev. D 66, 056006 (2002) [arXiv:hep-ph/0205314] and Phys. Rev. D 66, 036005 (2002) [arXiv:hep-ph/0204342]; T. G. Rizzo, Phys. Rev. D 64, 095010 (2001) [arXiv:hep$\mathrm{ph} / 0106336]$.

[4] See, for example, T.G. Rizzo and J.D. Wells, Phys. Rev. D61, 016007 (2000); P. Nath and M. Yamaguchi, Phys. Rev. D60, 116006 (1999); M. Masip and A. Pomarol, Phys. Rev. D60, 096005 (1999); L. Hall and C. Kolda, Phys. Lett. B459, 213 (1999); R. Casalbuoni, S. DeCurtis, D. Dominici and R. Gatto, Phys. Lett. B462, 48 (1999); 
A. Strumia, Phys. Lett. B466, 107 (1999); F. Cornet, M. Relano and J. Rico, Phys. Rev. D61, 037701 (2000); C.D. Carone, Phys. Rev. D61, 015008 (2000).

[5] T.G. Rizzo, Phys. Rev. D61, 055005 (2000) and Phys. Rev. D64, 015003 (2001).

[6] L. Randall and R. Sundrum, Phys. Rev. Lett. 83, 3370 (1999) [arXiv:hep-ph/9905221].

[7] For an overview of the Randall-Sundrum model phenomenology, see H. Davoudiasl, J.L. Hewett and T.G. Rizzo, Phys. Rev. Lett. 84, 2080 (2000); Phys. Lett. B493, 135 (2000); and Phys. Rev. D63, 075004 (2001).

[8] For a review of new gauge boson physics at colliders and details of the various models, see J.L. Hewett and T.G. Rizzo, Phys. Rep. 183, 193 (1989); M. Cvetic and S. Godfrey, in Electroweak Symmetry Breaking and Beyond the Standard Model, ed. T. Barklow et al., (World Scientific, Singapore, 1995), hep-ph/9504216; T.G. Rizzo in New Directions for High Energy Physics: Snowmass 1996, ed. D.G. Cassel, L. Trindle Gennari and R.H. Siemann, (SLAC, 1997), hep-ph/9612440; A. Leike, Phys. Rep. 317, 143 (1999).

[9] This is a common feature of the class of models wherein the usual $S U(2)_{L}$ of the SM is the result of a diagonal breaking of a product of two or more $S U(2)$ 's. For a discussion of a few of these models, see H. Georgi, E.E. Jenkins, and E.H. Simmons, Phys. Rev. Lett. 62, 2789 (1989) and Nucl. Phys. B331, 541 (1990); V. Barger and T.G. Rizzo, Phys. Rev. D41, 946 (1990); T.G. Rizzo, Int. J. Mod. Phys. A7, 91 (1992); R.S. Chivukula, E.H. Simmons and J. Terning, Phys. Lett. B346, 284 (1995); A. Bagneid, T.K. Kuo, and N. Nakagawa, Int. J. Mod. Phys. A2, 1327 (1987) and Int. J. Mod. Phys. A2, 1351 (1987); D.J. Muller and S. Nandi, Phys. Lett. B383, 345 (1996); X.Li and E. Ma, Phys. Rev. Lett. 47, 1788 (1981) and Phys. Rev. D46, 1905 (1992); E. Malkawi, T.Tait and C.-P. Yuan, Phys. Lett. B385, 304 (1996).

[10] See, for example, the analysis by G. Azuelos and G. Polesello in, G. Azuelos et al., "The beyond the standard model working group: Summary report," Proceedings of Workshop on Physics at TeV Colliders, Les Houches, France, 21 May - 1 Jun 2001, arXiv:hep-ph/0204031; T. G. Rizzo, in Proc. of the APS/DPF/DPB Summer Study on the Future of Particle Physics (Snowmass 2001) ed. N. Graf, eConf C010630, P304 (2001) [arXiv:hep-ph/0109179]. 
[11] F. Gianotti et al., arXiv:hep-ph/0204087.

[12] T. G. Rizzo, Phys. Rev. D 55, 5483 (1997) [arXiv:hep-ph/9612304].

[13] T. G. Rizzo, [arXiv:hep-ph/0303056]. 